\title{
ENDURECIMIENTO DE LA CAPA SUPERFICIAL DE ALEACIONES EN BASE DE COBRE POR EL TRATAMIENTO DE BORONIZADO
}

\author{
Responsable : Ing. Freddy Felipe Cori Nina. \\ Miembros : Ing. Julián Nieto Quispe. \\ Ing. Avelino Pari Pinto
}

RESUMEN

Se ha investigado la influencia de la capa de boruros depositada en la superficie de una aleación en base de cobre como el SAE 65, sobre el comportamiento al desgaste y la dureza. Los recubrimientos fueron obtenidos por el proceso de metalización de difusión e intercambio gas-metal, utilizando una mezcla de sales como carbón vegetal, mineral ulexita, ácido bórico y bórax, que proporcionan el elemento difundidor, mediante ensayos metalográficos, de dureza Vickers y según a un modelo experimental se determinó el espesor de la capa de boruros depositado; se seleccionó como parámetros de temperatura de recubrimiento a $840^{\circ} \mathrm{C}$ y un tiempo de 4.30 horas y con una mezcla de componentes determinado, mediante la ecuación utilizada en el modelo se obtuvo un espesor de capa deseada. Luego de los ensayos realizados se determinó que las muestras tratadas han incrementado su dureza y la resistencia al desgaste ligeramente en estas aleaciones.
ABSTRACT

The influence of the layer of boruros has been investigated, deposited in the surface of an alloy in copper base like SAE 65, on the behavior to the wear away and the hardness. The coverings were obtained by the process of bonding of diffusion and interchange gas - metal, having used a mixture of salts like vegetal coal, the ulexita mineral, the boric acid and the borax. that they provide the difundidor element. By means of metalograficos tests, of hardness Vickers and on the basis of an experimental model the thickness of the layer of boruros was determined deposited; it was selected as parameters of the temperature of covering to $840^{\circ} \mathrm{C}$ and a time of 4,30 hours and with a mixture of components determined, by means of the equation used in the model obtained a thickness of wished layer. After made tests one determined that the treated test tubes have increased their hardness and the resistance to the wear away down slightly in these alloys.

\section{INTRODUCCIÓN}

En Tarata, Tacna, existen afloraciones de ulexita y está en prospección, otro yacimiento en plena explotación está en la laguna de Salinas, Arequipa. La ulexita es un compuesto natural que contiene boratos. Los niveles de desarrollo económico y tecnológico de la producción nacional de minerales no - metálicos son muy bajos en comparación con la mineria metálica; esto ocasiona una notable dependencia económica del sector industrial de no-metálicos con respecto a la importación de estos insumos.

Es oportuno revisar y dirigir la atención sobre aquellos recursos naturales que existen en nuestro territorio, los cuales no son aprovechados adecuadamente, como es el caso de los minerales no- metálicos de uso industrial. En la actualidad, los productos basados en boro son usados en una gran variedad de aplicaciones industriales y productos de consumo. El boro se encuentra en la naturaleza como un constituyente menor de muchos minerales como ulexita ( $\mathrm{NaCaB} \mathrm{O}_{5} 8 \mathrm{H}_{2} \mathrm{O}$ ), la colemanita $\left(\mathrm{Ca}_{2} \mathrm{~B}_{6} \mathrm{O}_{11} 5 \mathrm{H}_{2} \mathrm{O}\right)$, la kernita $\left(\mathrm{Na}_{2} \mathrm{~B}_{4} \mathrm{O}_{7} 4 \mathrm{H}_{2} \mathrm{O}\right)$ y la boracita $\left(\mathrm{Mg}_{7} \mathrm{C}_{12} \mathrm{~B}_{16} \mathrm{O}_{30}\right)$ de donde se obtiene ácido bórico $\mathrm{H}_{3} \mathrm{BO}_{4}$ y bórax $\left(\mathrm{Na}_{2} \mathrm{~B}_{4} \mathrm{O}_{7} 10 \mathrm{H}_{2} \mathrm{O}\right)$ yen el agua de mar, unas pocas partes por millón. La gran mayoría de boratos se han originado por deposición en lagos salinos, siendo los depósitos económicamente beneficiables los que se producen en las orillas y fondo de los lagos. EL boro es un elemento metaloide, semiconductor, trivalente que existe abundantemente en el mineral bórax. Hay dos alótropos del boro: el boro amorfo es un polvo marrón, pero el boro metálico es negro. La forma metálica es dura (9,3 en la escala de Mohs) y es un mal conductor a temperatura ambiente; pero es buen conductor de la electricidad a altas temperaturas. Este metaloide tiene la más alta resistencia a la tracción entre los elementos quimicos conocidos; el material fundido con arco tiene una resistencia mecánica entre 1600 y $2400 \mathrm{MPa}$. Entre muchas aplicaciones, el boro tiene además cualidades lubricantes similares al grafito y comparte con el carbono la capacidad de formar redes moleculares mediante enlaces covalentes estables, es mucho más parecido al carbono y al silicio en sus propiedades químicas. En metalurgia es utilizado para recubrimientos superficiales de aleaciones metálicas.

Los bronces $\mathrm{Cu}$-Sn tiene sus propiedades especiales influidas por el estaño; tienen buena resistencia a la corrosión en atmósferas industriales y marinas, son resistentes al desgaste y tienen fuerte resistencia mecánica. El bronce SAE-65 es una aleación antifricción con una resistencia a la tracción de $25 \mathrm{~kg} / \mathrm{mm}^{2}$ Las aplicaciones más comunes son en la fabricación de coronas, engranajes, cojinetes y tornillos sin fin.

La investigación nos permite conocer la influencia de las variables como el tiempo y la temperatura sobre la dureza y la resistencia al desgaste en relación con el espesor de la capa obtenida luego del tratamiento que permite relativamente una mejora en las aplicaciones de piezas combinadas de acero-bronce con movimiento relativo. La hipótesis del estudio es "La dureza y resistencia al desgaste de la aleación de base de cobre SAE-65 aumentará con el 
tratamiento térmico de recubrimiento superficial con el boro". Para la evaluación de los resultados se optó por el diseño factorial y ayuda del software Statgraphics plus versión 4, que relaciona las variables del espesor de la capa, temperatura y el tiempo.

\section{MATERIAL Y MÉTODOS}

El procedimiento a seguir ha sido la preparación metalográfica de las probetas y la evaluación de sus caracteristicas, pero inicialmente sin considerar el tratamiento; luego las probetas son sometidas al tratamiento de metalización por difusión con boro y su respectiva evaluación final.

La mezcla I seleccionada para la producción de gas de difusión tiene la composición siguiente: $5 \%$ de mineral de ulexita $\left(\mathrm{NaCaB}, \mathrm{O}_{0} 8 \mathrm{H}_{2} \mathrm{O}\right.$ ) procedente de la laguna de Salinas (Arequipa), $25 \%$ de ácido bórico $\left(\mathrm{H}_{3} \mathrm{BO}_{4}\right), 5 \%$ de bórax $\left(\mathrm{Na}_{2}\right.$ $\mathrm{B}_{4} \mathrm{O}_{7} 10 \mathrm{H}_{2} \mathrm{O}$ ), $35 \%$ de carbón vegetal (olivo y huarango) con un granulado de 3 a $9 \mathrm{~mm}$; también se agregó arena y arcilla en un $30 \%$ para uniformizar la mezcla.La composición quimica nominal según la norma SAE para el bronce SAE65 se indica en la Tabla $\mathrm{N}^{\circ} 2-5$.

Tabla № 2-5 Composición Química del Bronce Sae-65

\begin{tabular}{|c|c|c|c|c|c|c|c|c|c|}
\hline SAE & Cu & Sn & Pb & Zn & Ni & P & Fe & Al & Mn \\
\hline 65 & $88-90$ & $10-12$ & 0,5 & 0,5 & 1 máx & $0,1-0,3$ & 0,15 & 0,005 & - \\
\hline
\end{tabular}

Fuente: Databook American Society for Metals 1999

El bronce SAE 65 tiene, según la norma SAE, una resistencia a la tracción nominal de $25 \mathrm{~kg} / \mathrm{mm}^{2}$, un alargamiento del $10 \%$, la dureza Brinell de 75 ; sus aplicaciones principales son aptas para engranajes, tornillos sinfin, tuercas y placas dentadas, placas de fricción. Los principales equipos utilizados han sido; un horno de mufla de $0{ }^{a} \mathrm{C}$ á $1000{ }^{\circ} \mathrm{C}$ de temperatura, además crisoles de cerámica, un microscopio metalográfico NEOPHOT 21, un microscopio metalográfico marca Metavar, lijas de agua $\mathrm{N}^{\circ} \mathrm{s}$ : $100,240,320 ; 400$ y 600 , paños de lana para pulido grueso y fino, un equipo de ensayo Universal de 10 toneladas de procedencia Hủngara, para los ensayos de tracción.

\section{1) Principales Ensayos a Probetas sin Tratamiento}

Se han preparado para estudio metalográfico 24 probetas de bronce SAE- 65 , de $1.5 \times 2 \times 3 \mathrm{~mm}$, de las que se realizaron los ensayos micrográficos, dureza, y microdureza, de las probetas sin recubrimiento.

Análisis químico de la muestra. El análisis quimico del bronce SAE-65 utilizado en el proyecto se realizó en la empresa de la Fundición NEPTUNO (Chile) y se muestra en la Tabla N ${ }^{0} 3-2$ siguiente:

Tabla N ${ }^{\circ}$ 3-2: Composición Quimica del Bronce Sae-65 Utilizado en el Proyecto (\%).

\begin{tabular}{|c|c|c|c|c|c|c|c|c|c|c|}
\hline Muestra & $\mathrm{Cu}$ & $\mathrm{Sn}$ & $\mathrm{Zn}$ & $\mathrm{Pb}$ & $\mathrm{Ni}$ & $\mathrm{Fe}$ & $\mathrm{Si}$ & $\mathrm{Al}$ & $\mathrm{P}$ & $\mathrm{Mn}$ \\
\hline SAE-65 & 86,70 & 12.06 & 0.60 & 0.496 & 0.003 & 0.016 & 0.045 & 0.004 & 0.013 & 0.067 \\
\hline
\end{tabular}

Ensayo de Tracción. Para los ensayos de tracción se ejecutó de acuerdo con las dimensiones establecidas en la norma técnicaASTME8 M y los resultados son 21.57 $\mathrm{kg} / \mathrm{mm}^{2}$ de resistencia a la tracción y de $823 \%$ de elongación.
Ensayo de Dureza.- Las probetas pulidas (04) al espejo de la aleación SAE-65 sin tratamiento se ensayaron en un microdurómetro Vickers marca Mitutoyo Hardmatic; la primera lectura a los $0.1 \mathrm{~mm}$ de distancia del borde y las siguientes cada $.8 \mathrm{~mm}$ de distancia, Se realizaron 8 imprentas en cada muestra, los promedios de dureza de las 04 probetas se muestran en la Tabla Nº $3-5$.

Tabla $N^{\circ} 3-5$ : Medición de durezas promedio del bronce SAE 65 sin tratamiento

\begin{tabular}{|c|c|c|c|c|c|c|c|c|c|}
\hline \multirow{8}{*}{ Muestra } & \multicolumn{7}{|c|}{ Mediciones de dureza Vickers } & \multicolumn{2}{|c|}{$\sum$} \\
\cline { 2 - 10 } & 0,1 & 0,9 & 1,7 & 2,5 & 3,3 & 4,1 & 4,9 & 5,7 & - \\
\hline 1 A & 89 & 80 & 95 & 104 & 96 & 94 & 89 & 87 & 91.75 \\
\hline
\end{tabular}

Ensayo de desgaste.-Para el ensayo se han considerado las características siguientes: método utilizado rozamiento puro; carga constante utilizada 24 kg; velocidad del torno con la probeta 380 RPM; en seco y sin lubricación; el tiempo considerado es de 2 horas; las dimensiones de la probeta de bronce SAE-65 a ensayarse es de $23 \mathrm{~mm}$ de diámetro externo, $12,7 \mathrm{~mm}$ de diámetro menor con una longitud de $10 \mathrm{~mm}$ en el punto de contacto con el eje de acero. Los resultados del ensayo nos dan $6,82 \%$ de desgaste.

\section{2) Análisis Metalográfico}

La fotomicrografía se tomó con una cámara digital Casio y se presenta en la Figuras $N^{0} 3.2$ muestra (a) observada a $26 x$ (aumentos) y (b) a 50x (aumentos), respectivamente; se ve que la estructura corresponde a una solución sólida alfa $a, y$ eutectoide alfa más delta $\delta$ ( áreas claras). Se observa una clara segregación quimica por efecto de la solidificación dendrítica.
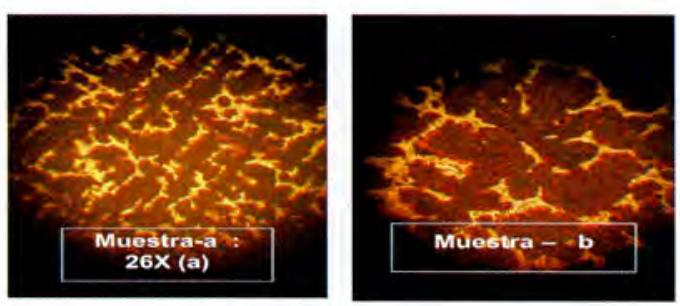

Figura $\mathrm{N}^{\circ}$ 3.2: Estructuras del bronce SAE-65,. particulas de $\delta$ en una matriz de a ( a y b es la misma probeta a diferentes aumentos).

\section{3) Metodología}

Luego de evaluadas las caracteristicas de las probetas sin tratamiento; se procedió a introducir las muestras en crisoles de cerámica envueltas dentro de la mezcla y cerradas herméticamente para evitar la fuga de gases de los recipientes. Los crisoles asi sellados, se han introducido al horno a la temperatura entre 800 y $880^{\circ} \mathrm{C}$ a un tiempo entre 3 á 6 horas respectivamente. Luego del tratamiento las muestras son preparadas para los análisis correspondiente. Finalmente se han introducido los datos al modelo seleccionado para con los resultados hacer 3 repeticiones de ensayos a $840^{\circ} \mathrm{C}$ y 4.30 horas, dando como resultado una ecuación con las variables tiempo, temperatura y espesor de la capa. 


\section{RESULTADOS Y DISCUSIÓN}

1) Determinación de las Condiciones de Tiempo y Temperatura de Tratamiento según el Modelo Superficie Respuesta.

Con los datos del análisis de las muestras de repetición y aplicando el modelo estadístico que relacionat, Ty el espesor de la capa $X$, de tratamiento de metalización por difusión del boro, con estos resultados se determinó el modelo matemático para el cálculo del espesor del la capa depositada y que se expresa en el $N^{0}$ de Figura 4.2. Las relaciones de ajuste son las siguientes:

Espesor $=14,1543-0,01675^{*}$ Temperatura-3,96 * Tiempo + 0,00483333 * Temperatura * Tiempo.

Donde:

Constante

$=14,1543$.

A: temperatura

$=-0,01675$

B: Tiempo

$=-3,96$

AB

$=0,00483333$

Contornos de Superficie de la Respuesta Estimada

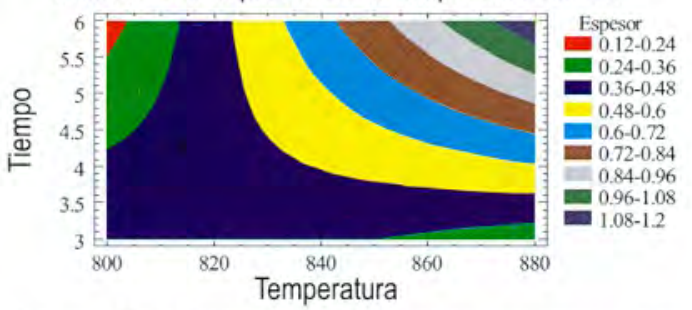

Figura $\mathrm{N}^{\circ}$ 4.2: Curva del espesor de isovalores de la capa en función del el tiempo, la temperatura de y del tratamiento respectivo.

En la Figura 4.2, se ha realizado una representación de la superficie respuesta mediante la curva de isovalores donde se puede localizar las combinaciones de valores del tiempo, la temperatura y el espesor de la capa correspondiente, puede apreciarse que para la temperatura $(T)$ de $840{ }^{\circ} \mathrm{C}$ y un tiempo $(t)$ de 4,30 horas resulta aproximadamente en la zona de color amarillo que corresponde a espesores de 0,48 á 0,6 mm. Esta representación ayuda a buscar el tratamiento óptimo en cuanto a espesores, sin olvidar que no se conoce la calidad de la capa formada en las condiciones elegidas.

\section{2) Ensayo de Probetas de la Aleación SAE-65 con Recubrimiento.}

1. Ensayo de Microdureza Vickers .- Estos ensayos se realizaron con un microdurómetro marca Mitutoyo Hardmatic. Las durezas se tomaron del borde hacia el núcleo, la primera lectura a $0.1 \mathrm{~mm}$ de distancia del borde y las siguientes cada $0.8 \mathrm{~mm}$, en total 8 lecturas por muestra ( Tab. 4-1).

Tabla N 4-1: Medición de durezas promedio del bronce SAE 65 con tratamiento

\begin{tabular}{|c|c|c|c|c|c|c|c|c|}
\hline \multirow{2}{*}{ Muestra } & \multicolumn{8}{|c|}{$\begin{array}{c}\text { Mediciones de dureza Vickers a probetas } \\
\text { con tratamiento }\end{array}$} \\
\cline { 2 - 9 } & 0,1 & 0,9 & 1,7 & 2,5 & 3,3 & 4,1 & 4,9 & 5,7 \\
\hline$\sum$ & 114 & 119 & 111 & 96 & 89 & 80 & 70 & 68 \\
\hline
\end{tabular}

2. Ensayo de Desgaste.- Estos ensayos se efectuaron en las mismas condiciones que para las probetas sin tratamiento. Los resultados como promedio, han sido de $5,05 \%$ de desgaste.

3. Ensayo de Tracción. El ensayo de las muestras tratadas aumentó muy levemente dando como resultado $22,12 \mathrm{~kg} / \mathrm{mm} 2$.

4. Ensayo Micrográfico a Probetas con Tratamiento
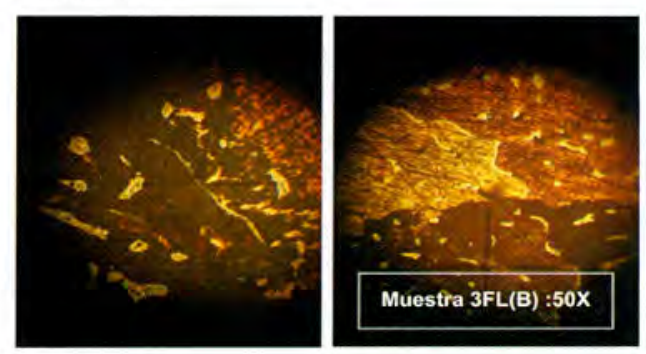

Fig. $N^{\circ}$ 4-1 Microestructura del bronce SAE-65 con Tratamiento

\begin{abstract}
La fase a, que es rica en cobre, es maleable en frio, mientras que la fase $\delta$ es dura; debido al tratamiento se originaron boruros resistentes al desgaste y de gran fragilidad; por lo que es necesario un tratamiento de homogenización.
\end{abstract}

\section{3) Resultados}

De los ensayos realizados para el presente trabajo, se puede concluir que "la dureza y resistencia al desgaste de la aleación SAE-65, aumenta con el tratamiento termoquímico por difusión de boro; que corrobora la afirmación indicada en la hipótesis. Los principales resultados se muestran en la Tabla $N^{0}$ 4-5.

Tabla N 4-5: Resultado de los ensayos realizados a las probetas de bronce SAE-65.

\begin{tabular}{|l|c|c|}
\hline Caracteristicas & Sin tratamiento & Con tratamiento \\
\hline Ensayo de dureza & $91,75 \mathrm{HV}$ (Vickers) & $114 \mathrm{HV}$ \\
\hline Ensayo de desgaste & $6.82 \%$ & $5.06 \%$ \\
\hline Ensayo de tracción & $21,57 \mathrm{Kg} / \mathrm{mm} 2)$ & $22,12 \mathrm{Kg} / \mathrm{mm} 2)$ \\
\hline
\end{tabular}

Donde la dureza del bronce SAE-65 en promedio y sin tratamiento fue de $91,75 \mathrm{HV}$; pero al ser tratadas su dureza se ha incrementado a 114 y 119 HV en la superficie tratada. Asimismo, el desgaste en las probetas $\sin$ tratamiento ha sido del $6,82 \%$ de desgaste; pero en las probetas con tratamiento disminuyó a $5,06 \%$. En cuanto a la resistencia a la tracción fue de $21,57 \mathrm{~kg} / \mathrm{mm} 2$ ; después del tratamiento se incremento muy levemente a 22,12 casino se haincrementado.

\section{CONCLUSIONES}

1. Con respecto al bronce SAE-65, la mayor dureza de la capa fue obtenida con la mezcla I, con un valor de 114 y $119 \mathrm{HV}$, que es mucho más alto que el indicado por la probeta sin tratamiento $91,75 \mathrm{HV}$. Como comparación de sus caracteristicas de resistencia a la tracción debido al tratamiento esta también se elevó muy levemente. Asimismo, debido al aumento de su dureza el desgaste, 
es menor en probetas tratadas. El modelo utilizado nos da una ecuación para obtener un espesor deseado considerando 4,30 horas y a $840^{\circ} \mathrm{C}$ de tratamiento, obteniéndose un espesor de 0,48 0,6 mm. "La dureza y la resistencia al desgaste de la aleación en base de cobre SAE-65 aumenta con el tratamiento de metalización superficial con et boro".

2. Todos los tratamientos generaron una capa, en mayor o menor grado, lo que prueba el fenómeno de difusión en el latón tratado. Al concluirse el presente indicamos que hubiera sido deseable un mayor número de experiencias basadas en mayor número de probetas y ensayos respectivamente.

\section{BIBLIOGRAFÍA}

A. M. Afanásiev-V. A. Marien, Prácticas de laboratorio de resistencia de materiales, MIR Editorial, Moscú 1978; 42,43 .
E. Ponce, Tratamientos Térmicos Experimentales con Boro, Congreso Internacional Metalurgia, UNSA 1999. Perú.

J. C. Anderson, K., D. Leaver, Ciencia del los Materiales, $2^{\circ}$ ediciones, LIMUSA editorial, México 2002. 273, 278.

Asdrúbal Valencia, Tecnologia del tratamiento térmico de los metales, Universidad editorial de Antioquia, $2^{\circ}$ Edición, Colombia, 1992.357.

Avner S. (1991), Introducción a la metalúrgia fisica fisica" Editorial Mc.Graw, Hill, México. 317-325pp.

Bokstein B. S. (1980) "Difusión de metales"Editorial MirMoscú Editorial.

[32] Carl A. Keyser; Técnicas de laboratorio para pruebas de materiales, Edi, LIMUSA, Mexico, 19965; 49.

Donald R. Askeland, La ciencia e ingenieria de los materiales, Grupo Editorial Iberoamericana, México 1987; $110,498-49$.

A. P. Guliáev, Metalografia, Tomo I, MIR Editorial-Moscú, URSS, 1978. (p.334). 